\title{
STRONGLY EXPOSED POINTS IN LEBESGUE-BOCHNER FUNCTION SPACES
}

\author{
ZHIBAO HU AND BOR-LUH LIN
}

(Communicated by Dale Alspach)

\begin{abstract}
It is a result of Peter Greim that if $f$ is a strongly exposed point of the unit ball of Lebesgue-Bochner function space $L^{p}(\mu, X), 1<p<\infty$, then $f$ is a unit vector and $f(t) /\|f(t)\|$ is a strongly exposed point of the unit ball of $X$ for almost all $t$ in the support of $f$.

We prove that the converse is also true.
\end{abstract}

Throughout we assume that $X$ is a Banach space, $1<p, q<\infty$ with $1 / p+1 / q=1$, and $(\Omega, \Sigma, \mu)$ is a positive measure space. We use $S_{X}$ and $B_{X}$ to denote the unit sphere and the unit ball in $X$ respectively and use $\mu^{*}$ to denote the outer measure associated with $\mu$. For a subset $K$ of $X$ we use str-exp $K$ to denote the set of strongly exposed points of $K$. Recall that $x$ is a strongly exposed point of $K$ if $x \in K$ and there exists $x^{*}$ in $X^{*}$ which strongly exposes $K$ at $x$, that is, $\sup x^{*}(K)=x^{*}(x)$ and, whenever $\left\{x_{n}\right\} \subset K$ and $\lim _{n} x^{*}\left(x_{n}\right)=x^{*}(x), \lim _{n} x_{n}=x$.

Johnson [J] and Greim [G1, G2] studied the strongly exposed points in Lebesgue-Bochner function spaces $L^{p}(\mu, X)$. In [G1, G2], it is shown that, for $f \in \operatorname{str}-\exp B_{L^{p}(\mu, X)}$, it is necessary and, in case $X$ is smooth, is also sufficient that, for almost all $t$ in the support of $f(\operatorname{supp} f), f(t) /\|f(t)\| \in \operatorname{str}-\exp B_{X}$ and $\|f\|=1$. The purpose of this note is to show that these conditions are sufficient in general (Theorem 7).

Strongly exposed points can be described in terms of slices. For $K \subset X$, the slice of $K$ determined by the functional $x^{*}$ in $X^{*}$ and $\delta>0$ is the subset of $K$ given by

$$
S\left(x^{*}, K, \delta\right)=\left\{x \in K: x^{*}(x)>\sup x^{*}(K)-\delta\right\} .
$$

It is obvious that $x^{*}$ strongly exposes $K$ at $x$ if and only if for any $\varepsilon>0$ there is $\delta>0$ such that $\operatorname{diam} S\left(x^{*}, K, \delta\right)<\varepsilon$ and $x \in S\left(x^{*}, K, \delta\right)$, where $\operatorname{diam} S\left(x^{*}, K, \delta\right)$ is the diameter of the set $S\left(x^{*}, K, \delta\right)$.

The key to our discussion is the following characterization of strongly exposed points "exclusively" in terms of slices (i.e., it does not use strongly exposing functionals): an element $x$ in $K$ is a strongly exposed point of $K$ if and only if there exist $\varepsilon_{n}>0$ and $\delta_{n}>0$, with $\lim _{n} \varepsilon_{n}=0$ and $\lim _{n} \delta_{n}=0$, and

Received by the editors April 10, 1992 and, in revised form, July 21, 1992.

1991 Mathematics Subject Classification. Primary 46B20, 46E40.

Key words and phrases. Lebesgue-Bochner function space, strongly exposed point, slice. 
a bounded sequence $\left\{x_{n}^{*}\right\} \subset X^{*}$, such that $x$ belongs to each $S\left(x_{n}^{*}, K, \delta_{n}\right)$ and $\operatorname{diam} S\left(x_{n}^{*}, K, \delta_{m}\right) \leq \varepsilon_{m}$ for $n \geq m \geq 1$. Lemma 2 is a variation of this observation for Lebesgue-Bochner function spaces.

Lemma 1. Let $f \in L^{p}(\mu)$ such that $f(t) \geq 0$ for all $t$ in $\Omega$. If $\left\{f_{\lambda}\right\}$ is a net in $L^{p}(\mu, X)$ such that $\left\{\left\|f_{\lambda}(\cdot)\right\|\right\}$ converges to $f$ in $L^{p}(\mu)$ then there is a net $\left\{g_{\lambda}\right\}$ in $L^{p}(\mu, X)$ such that $\left\|g_{\lambda}(t)\right\|=f(t)$, for all $t$ and $\lambda$, and $\lim _{\lambda}\left\|f_{\lambda}-g_{\lambda}\right\|=0$. Proof. Pick $x$ in $S_{X}$. For each $\lambda$, let $E_{\lambda}=\left\{t: t \in \Omega\right.$ and $\left.f_{\lambda}(t)=0\right\}$ and define

$$
g_{\lambda}(t)= \begin{cases}f(t) x, & t \in E_{\lambda}, \\ f(t) f_{\lambda}(t) /\left\|f_{\lambda}(t)\right\|, & t \notin E_{\lambda} .\end{cases}
$$

Then $\left\|g_{\lambda}(t)\right\|=f(t)$ and $\left\|f_{\lambda}(t)-g_{\lambda}(t)\right\|=\left|\left\|f_{\lambda}(t)\right\|-f(t)\right|$ for all $t$ and $\lambda$. Hence, $g_{\lambda} \in L^{p}(\mu, X)$ and $\lim _{\lambda}\left\|f_{\lambda}-g_{\lambda}\right\|=\lim _{\lambda}\|\| f_{\lambda}(\cdot)\|-f\|=0$. Q.E.D.

For each $f \in L^{p}(\mu, X)$, we denote by $S(f)$ the set

$\left\{g: g \in L^{p}(\mu, X)\right.$ and $\|g(t)\|=\|f(t)\|$ for all $t$ in $\left.\Omega\right\}$.

Lemma 2. Suppose $f \in S_{L^{p}(\mu, X)}$. Then the following are equivalent.

(1) $f \in \operatorname{str}-\exp B_{L^{p}(\mu, X)}$.

(2) There exist $\varepsilon_{n}>0$ and $\delta_{n}>0$, with $\lim _{n} \varepsilon_{n}=0$ and $\lim _{n} \delta_{n}=0$, and $\left\{T_{n}\right\}$ in $B_{L^{p}(\mu, X)^{*}}$ satisfying

$$
\begin{aligned}
& T_{n}(f)>1-\delta_{n}, \text { and for } m \leq n, \text { if } g \in S(f) \text { and } T_{n}(g)> \\
& 1-\delta_{m}, \text { then }\|f-g\| \leq \varepsilon_{m} .
\end{aligned}
$$

Moreover, if (2) is true, then every weak ${ }^{*}$ cluster point of $\left\{T_{n}\right\}$ strongly exposes $B_{L^{p}(\mu, X)}$ at $f$.

Proof. It is obvious that (1) implies (2). To prove the converse is true, let $T$ be a weak ${ }^{*}$ cluster point of $\left\{T_{n}\right\}$. Then $T(f)=1$ and $\|T\| \leq 1$. Thus

$$
\|T\|=1=T(f)=\sup T\left(B_{L^{p}(\mu, X)}\right) .
$$

Suppose $\left\{f_{n}\right\}$ is a sequence in $B_{L^{p}(\mu, X]}$ such that $\lim _{n} T\left(f_{n}\right)=1$. Then $\lim _{n} T\left(f_{n}+f\right)=2$. It follows that, in $L^{p}(\mu), \lim _{n}\|\| f_{n}(\cdot)\|+\| f(\cdot)\|\|=2$. By the uniform rotundity of $L^{p}(\mu)$, we have $\lim _{n}\left\|f_{n}(\cdot)\right\|=\|f(\cdot)\|$ in $L^{p}(\mu)$. Thus by Lemma 1 there is a sequence $\left\{g_{n}\right\}$ in $S(f)$ such that $\lim _{n}\left\|f_{n}-g_{n}\right\|=0$. It is obvious that $\lim _{n} T\left(g_{n}\right)=1$. For any $\varepsilon>0$, choose $m \geq 1$ such that $\varepsilon_{m}<\varepsilon$. There is $n_{1}>m$ such that for $k \geq n_{1}$ we have $T\left(g_{k}\right)>1-\delta_{m}$ and $\delta_{k}<\delta_{m}$. For each $k \geq n_{1}$, since $T$ is a weak* cluster point of $\left\{T_{n}\right\}$ and $T\left(g_{k}\right)>1-\delta_{m}$, there is $n \geq n_{1}$ such that $T_{n}\left(g_{k}\right)>1-\delta_{m}$. Thus $\left\|f-g_{k}\right\| \leq \varepsilon_{m}<\varepsilon$. Hence, $\lim _{n}\left\|g_{n}-f\right\|=0$. It follows that $\lim _{n}\left\|f_{n}-f\right\|=0$. Therefore, $T$ strongly exposes $B_{L^{p}(\mu, X)}$ at $f$. Q.E.D.

In the proof of Lemma 4 , we will construct a sequence $\left\{T_{n}\right\}$ like the one described in Lemma 2 , but our $\left\{T_{n}\right\}$ will be from $L^{q}\left(\mu, X^{*}\right)$, which is naturally a subspace of $L^{p}(\mu, X)^{*}$. Note that for any $\varphi \in L^{q}\left(\mu, X^{*}\right)$ and $h \in L^{p}(\mu, X)$ the action of $\varphi$ on $h$ is given by $(\varphi, h)=\int_{\Omega}(\varphi(t), h(t)) d \mu(t)$ [DU]. For the proof of Lemma 4 we need

Lemma 3. Suppose $(\Omega, \Sigma, \mu)$ is a probability space and $f \in B_{L^{p}(\mu, X)}$ with $f(\Omega) \subset B_{X}$. Let $0<\delta<1$ and $g: \Omega \rightarrow S_{X^{*}}$. If there are $\left\{t_{n}\right\} \subset \Omega$, $\left\{F_{n}\right\} \subset \Sigma$, and $r>0$ satisfying.

(1) diam $S\left(g\left(t_{n}\right), B_{X}, r\right)<\delta$ and $f\left(F_{n}\right) \subset S\left(g\left(t_{n}\right), B_{X}, r\right)$, and

(2) $F_{n} \cap F_{m}=\varnothing$ for $n \neq m$ and $\mu\left(\bigcup_{n} F_{n}\right)>1-2 \delta / 3$, 
then, for any measurable functions $\varphi: \Omega \rightarrow B_{X^{*}}$ and $h: \Omega \rightarrow B_{X}$ with $\left.\varphi\right|_{\bigcup_{n}} F_{n}=$ $\sum_{n} g\left(t_{n}\right) \chi_{F_{n}}$ and $\int_{\Omega}(\varphi(t), h(t)) d \mu(t) \geq 1-r \delta / 3$, we have $\|h-f\|<3 \delta^{1 / p}$.

Proof. Let $E_{n}=\left\{t: t \in F_{n}\right.$ and $\left.h(t) \notin S\left(g\left(t_{n}\right), B_{X}, r\right)\right\}$. Then $\|h(t)-f(t)\|<$ $\delta$ for $t \in F_{n} \backslash E_{n}$, and $\left(g\left(t_{n}\right), h(t)\right) \leq 1-r$ for $t \in E_{n}$. Since

$$
\begin{aligned}
1-\frac{r \delta}{3} & \leq \int_{\Omega}(\varphi(t), h(t)) d \mu(t) \\
& =\int_{\Omega \backslash \bigcup_{n} E_{n}}(\varphi(t), h(t)) d \mu(t)+\int_{\bigcup_{n} E_{n}}(\varphi(t), h(t)) d \mu(t) \\
& \leq \mu\left(\Omega \backslash \bigcup_{n} E_{n}\right)+\int_{\bigcup_{n} E_{n}}\left(\sum_{n} g\left(t_{n}\right) \chi_{F_{n}}, h(t)\right) d \mu(t) \\
& =\mu\left(\Omega \backslash \bigcup_{n} E_{n}\right)+\sum_{n} \int_{E_{n}}\left(g\left(t_{n}\right), h(t)\right) d \mu(t) \\
& \leq \mu\left(\Omega \backslash \bigcup_{n} E_{n}\right)+(1-r) \mu\left(\bigcup_{n} E_{n}\right)=1-r \mu\left(\bigcup_{n} E_{n}\right),
\end{aligned}
$$

we have $\mu\left(\bigcup_{n} E_{n}\right) \leq \delta / 3$. Thus

$$
\begin{aligned}
\|h-f\|^{p}= & \int_{\Omega \backslash \bigcup_{n} F_{n}}\|h(t)-f(t)\|^{p} d \mu(t)+\int_{\bigcup_{n} F_{n} \backslash E_{n}}\|h(t)-f(t)\|^{p} d \mu(t) \\
& +\int_{\bigcup_{n} E_{n}}\|h(t)-f(t)\|^{p} d \mu(t) \\
\leq & 2^{p} \mu\left(\Omega \backslash \bigcup_{n} F_{n}\right)+\delta^{p} \mu\left(\bigcup_{n} F_{n} \backslash E_{n}\right)+2^{p} \mu\left(\bigcup_{n} E_{n}\right) \\
\leq & 2^{p} 2 \delta / 3+\delta^{p}+2^{p} \delta / 3=2^{p} \delta+\delta^{p}<3^{p} \delta . \quad \text { Q.E.D. }
\end{aligned}
$$

If $(\Omega, \Sigma, \mu)$ is a probability space and $f \in B_{L^{p}(\mu, X)}$ with $f(\Omega) \subset$ str-exp $B_{X}$, then $f$ must be a strongly exposed point of $B_{L^{p}(\mu, X)}$ as shown in Lemma 4.

Lemma 4. Suppose $(\Omega, \Sigma, \mu)$ is a probability space and $f \in B_{L p(\mu, X)}$ with $f(\Omega) \subset S_{X}$. If there is a function $g: \Omega \rightarrow S_{X^{*}}$ such that, for every $t \in \Omega$, $g(t)$ strongly exposes $B_{X}$ at $f(t)$, then there exist $\varepsilon_{n}>0$ and $\delta_{n}>0$, with $\lim _{n} \varepsilon_{n}=0$ and $\lim _{n} \delta_{n}=0$, and $\left\{g_{n}\right\} \subset B_{L^{q}\left(\mu, X^{*}\right)}$ satisfying:

(1) $g_{n}(\Omega) \subset g(\Omega)$, and $\int_{\Omega}\left(g_{n}(t), f(t)\right) d \mu(t)>1-\delta_{n}$, and

(2) for $n \geq k \geq 1$, if $h \in S(f)$ and $\int_{\Omega}\left(g_{n}(t), h(t)\right) d \mu(t)>1-\delta_{k}$, then $\|f-h\| \leq \varepsilon_{k}$.

Thus, by Lemma 2, we have $f \in \operatorname{str}-\exp B_{L^{p}(\mu, X)}$.

Proof. We may assume that $f(\Omega)$ is separable. For $k \geq 1$ and $m \geq 1$ set $\alpha_{k}=2^{-k}$ and

$$
D(m, k)=\left\{t: t \in \Omega \text { and } \operatorname{diam} S\left(g(t), B_{X}, \frac{1}{m}\right)<\alpha_{k}\right\} .
$$

It is obvious that $D(m, k) \subset D(m+1, k)$. Since, for every $t \in \Omega, g(t)$ strongly exposes $B_{X}$ at $f(t)$, we have $\Omega=\bigcup_{m} D(m, k)$ for each $k \geq 1$. Thus 
for every subset $A$ of $\Omega$, we have

$$
\mu^{*}(A)=\lim _{m} \mu^{*}(A \cap D(m, k)) .
$$

In particular, there is $m_{1} \geq 1$ such that $\mu^{*}\left(D\left(m_{1}, 1\right)\right)>1-\alpha_{1} / 3$. Choose a measurable set $E(1,1)$ such that $E(1,1) \supset D\left(m_{1}, 1\right)$ and $\mu(E(1,1))=$ $\mu^{*}\left(D\left(m_{1}, 1\right)\right)$. Let $E(1,2)=\Omega \backslash E(1,1)$. Then $\{E(1,1), E(1,2)\}$ is a partition of $\Omega$.

Assume, for $1 \leq k \leq n$, we have chosen $m_{1}<\cdots<m_{n}$ and partitions $\{E(k, 1), E(k, 2), \ldots, E(k, k), E(k, k+1)\}$ of $\Omega$ so that, for $1 \leq i \leq k$,

(1) $\mu(E(k, i))=\mu^{*}(A(k, i))$

$(* *)$

$$
\text { where } A(k, i)=E(k, i) \cap\left(\bigcap_{j=i}^{k} D\left(m_{j}, j\right)\right) \text {, and }
$$

(2) $\sum_{j=1}^{i} \mu(E(k, j))>1-\frac{\alpha_{i}}{3}$.

Since $\sum_{j=1}^{i} \mu^{*}(A(n, j))>1-\alpha_{i} / 3$ and $\mu(E(n, n+1))+\sum_{j=1}^{n} \mu^{*}(A(n, j))$ $=1$, by $(*)$ there is $m_{n+1}>m_{n}$ such that

$$
\sum_{j=1}^{i} \mu^{*}\left(A(n, j) \cap D\left(m_{n+1}, n+1\right)\right)>1-\frac{\alpha_{i}}{3} \text { for } 1 \leq i \leq n
$$

and

$\mu^{*}\left(E(n, n+1) \cap D\left(m_{n+1}, n+1\right)\right)+\sum_{j=1}^{n} \mu^{*}\left(A(n, j) \cap D\left(m_{n+1}, n+1\right)\right)>1-\frac{\alpha_{n+1}}{3}$.

Let $A(n+1, n+1)=E(n, n+1) \cap D\left(m_{n+1}, n+1\right)$, and let $A(n+1, j)=$ $A(n, j) \cap D\left(m_{n+1}, n+1\right)$ for $1 \leq j \leq n$. Then, for $1 \leq j \leq n+1$, we can choose a measurable set $E(n+1, j)$ such that

$A(n+1, j) \subset E(n+1, j) \subset E(n, j)$ and $\mu(E(n+1, j))=\mu^{*}(A(n+1, j))$.

Let $E(n+1, n+2)=\Omega \backslash \bigcup_{j=1}^{n+1} E(n+1, j)$. Then $\{E(n+1,1), \ldots$, $E(n+1, n+1), E(n+1, n+2)\}$ is a partition of $\Omega$ which satisfies (**) for $1 \leq i \leq k=n+1$. By induction, there are natural numbers $m_{1}<m_{2}<\cdots$ and partitions $\{E(k, 1), \ldots, E(k, k+1)\}$ of $\Omega$ such that $(* *)$ is true for $1 \leq i \leq k \leq n<\infty$.

Now fix $n \geq 1$. It is obvious that $f(A(n, k)) \subset \bigcup\left\{S\left(g(t), B_{X}, \alpha_{n} / 3 m_{n}\right): t \in\right.$ $A(n, k)\}$ for $1 \leq k \leq n$. Since $\left\{S\left(g(t), B_{X}, \alpha_{n} / 3 m_{n}\right): t \in A(n, k)\right\}$ is an open covering of $f(A(n, k))$ which is separable, there is a sequence $\left\{t_{n k}^{j}\right\}_{j \geq 1}$ in $A(n, k)$ such that

$$
f(A(n, k)) \subset \bigcup_{j} S\left(g\left(t_{n k}^{j}\right), B_{X}, \frac{\alpha_{n}}{3 m_{n}}\right) .
$$

Define

$$
\begin{aligned}
& E(n, k, j) \\
& \quad=E(n, k) \cap f^{-1}\left\{S\left(g\left(t_{n k}^{j}\right), B_{X}, \frac{\alpha_{n}}{3 m_{n}}\right) \backslash \bigcup_{i<j} S\left(g\left(t_{n k}^{i}\right), B_{X}, \frac{\alpha_{n}}{3 m_{n}}\right)\right\} .
\end{aligned}
$$


Then $E(n, k, j)$ is measurable, and $E(n, k, j) \cap E\left(n, i, j^{\prime}\right)=\varnothing$ if $i \neq k$ or $j \neq j^{\prime}$. Since $A(n, k) \subset \bigcup_{j} E(n, k, j) \subset E(n, k)$ and $\mu(E(n, k))=$ $\mu^{*}(A(n, k))$, we have

$(* * *)$

$$
\mu\left(\bigcup_{j \geq 1} E(n, k, j)\right)=\mu(E(n, k)) .
$$

Thus by $(* *)$ we have $\mu(\bigcup\{E(n, k, j): 1 \leq k \leq n$ and $j \geq 1\})>1-\alpha_{n} / 3$. By definition, for all $t$ in $E(n, k, j)$ we have $\left(g\left(t_{n k}^{j}\right), f(t)\right)>1-\alpha_{n} / 3 m_{n}$. Hence,

$$
\begin{aligned}
& \left(\sum_{1 \leq k \leq n, j \geq 1} g\left(t_{n k}^{j}\right) \chi_{E(n, k, j)}, f\right) \\
& \quad>\left(1-\frac{\alpha_{n}}{3 m_{n}}\right) \mu(\bigcup\{E(n, k, j): 1 \leq k \leq n \text { and } j \geq 1\}) .
\end{aligned}
$$

Since, for each $t$ in $\Omega,(g(t), f(t))=1$, there is a measurable function $g_{n}: \Omega \rightarrow g(\Omega)$ such that

$$
\left.g_{n}\right|_{\bigcup_{1 \leq k \leq n, j \geq 1} E(n, k, j)}=\sum_{1 \leq k \leq n, j \geq 1} g\left(t_{n k}^{j}\right) \chi_{E(n, k, j)}
$$

and

$$
\left(g_{n}, f\right)=\int_{\Omega}\left(g_{n}(t), f(t)\right) d \mu(t)>1-\frac{\alpha_{n}}{3 m_{n}} .
$$

Let $\varepsilon_{n}=3\left(\alpha_{n}\right)^{1 / p}$ and $\delta_{n}=\alpha_{n} / 3 m_{n}$. To complete the proof it remains to verify the following claim.

Claim. For $n \geq k \geq 1$, if $h \in S(f)$ and $\int_{\Omega}\left(g_{n}(t), h(t)\right) d \mu(t)>1-\delta_{k}$, then $\|f-h\| \leq \varepsilon_{k}$.

For $1 \leq i \leq k$ and $j \geq 1$, since $t_{n i}^{j} \in A(n, i) \subset D\left(m_{k}, k\right)$ (see $\left.(* *)(1)\right)$, we have that

$$
\operatorname{diam} S\left(g\left(t_{n i}^{j}\right), B_{X}, 1 / m_{k}\right)<\alpha_{k} .
$$

By $(* *)$ and $(* * *)$, we have

$$
\begin{array}{r}
\mu(\bigcup\{E(n, i, j): 1 \leq i \leq k \text { and } j \geq 1\}) \\
=\sum_{i=1}^{k} \mu(E(n, i))>1-\frac{\alpha_{k}}{3}>1-\frac{2 \alpha_{k}}{3} .
\end{array}
$$

It is obvious that

$$
f(E(n, i, j)) \subset S\left(g\left(t_{n i}^{j}\right), B_{X}, \alpha_{n} / 3 m_{n}\right) \subset S\left(g\left(t_{n i}^{j}\right), B_{X}, 1 / m_{k}\right)
$$

and

$$
\left.g_{n}\right|_{1 \leq i \leq k, j \geq 1} E(n, i, j)=\sum_{1 \leq i \leq k, j \geq 1} g\left(t_{n i}^{j}\right) \chi_{E(n, i, j)} .
$$

By Lemma 3, we can conclude that, if $h \in S(f)$ and $\int_{\Omega}\left(g_{n}(t), h(t)\right) d \mu(t)>$ $1-\alpha_{k} / 3 m_{k}$, then $\|f-h\| \leq 3\left(\alpha_{k}\right)^{1 / p}$; in other words, if $h \in S(f)$ and $\int_{\Omega}\left(g_{n}(t), h(t)\right) d \mu(t)>1-\delta_{k}$, then $\|f-h\| \leq \varepsilon_{k}$. Thus the claim holds. Q.E.D.

The general case can be reduced to special case in Lemma 4 by using Lemmas 5 and 6. 
Lemma 5. Suppose $(\Omega, \Sigma, \mu)$ is a positive finite measure space and $f \in S_{L^{p}(\mu, X)}$, and suppose there is $M>0$ such that $1 / M \leq\|f(t)\| \leq M$ for all $t$ in $\Omega$. Let $f_{0}(t)=f(t) /\|f(t)\|$ for $t \in \Omega$, and let $\mu_{0}=\mu / \mu(\Omega)$. Then $f \in \operatorname{str}-\exp B_{L^{p}(\mu, X)}$ if and only if $f_{0} \in \operatorname{str}-\exp B_{L^{p}\left(\mu_{0}, X\right)}$.

Proof. Suppose $f_{0} \in \operatorname{str}-\exp B_{L^{p}\left(\mu_{0}, X\right)}$. Then there is $T \in S_{L^{p}\left(\mu_{0}, X\right)^{*}}$ which strongly exposes $B_{L^{p}\left(\mu_{0}, X\right)}$ at $f_{0}$. There is a function $g$ from $\Omega$ to $X^{*}$ such that $g$ is weak ${ }^{*}$ measurable (that is, for every $x$ in $X$ the real-valued function $g(\cdot)(x)$ is measurable), $\|g(\cdot)\| \in S_{L^{q}\left(\mu_{0}\right)}$, and for any $\varphi \in L^{p}(\mu, X)$ [EV, IT],

$$
T(\varphi)=\int_{\Omega}(g(t), \varphi(t)) d \mu(t) .
$$

By Theorem 2 [G2], we have $\|g(t)\|=1$ and $g(t)$ strongly exposes $B_{X}$ at $f_{0}(t)$ for almost all $t$ in $\Omega$. Let $h(t)=\|f(t)\|^{p-1} g(t)$ for all $t$ in $\Omega$. Then $\|h(\cdot)\|$ strongly exposes $B_{L^{p}(\mu)}$ at $\|f(\cdot)\|$, and $h(t)$ strongly exposes $B_{X}$ at $f(t) /\|f(t)\|$ for almost all $t$ in $\Omega$. By Theorem $2[\mathrm{G} 2]$ the functional in $L^{p}(\mu, X)^{*}$ represented by $h$ strongly exposes $B_{L^{p}(\mu, X)}$ at $f$.

The proof of the converse is similar. Q.E.D.

Lemma 6 (see [S, Theorem, p. 154]). Suppose $\left\{X_{i}\right\}_{i \in I}$ is a family of Banach spaces. Let $f=(f(i))_{i \in I} \in l^{p}\left(X_{i}\right)$. Then $f$ is a strongly exposed point of the unit ball of $l^{p}\left(X_{i}\right)$ if and only if $\|f\|=1$ and $f(i) /\|f(i)\| \in \operatorname{str}-\exp B_{X_{i}}$ for every $i \in \operatorname{supp} f$.

Theorem 7. Suppose $f$ is an element in $L^{p}(\mu, X)$. If $\|f\|=1$ and $f(t) /\|f(t)\|$ $\in \operatorname{str}-\exp B_{X}$ for almost all $t \in \operatorname{supp} f$, then $f \in \operatorname{str}-\exp B_{L^{p}(\mu, X)}$.

Proof. Let $E_{0}=f^{-1}(0)$, and, for each integer $n \geq 1$, let

$$
E_{2 n}=\left\{t: t \in \Omega, 2^{n-1}<\|f(t)\| \leq 2^{n}\right\}
$$

and

$$
E_{2 n-1}=\left\{t: t \in \Omega, 2^{-n}<\|f(t)\| \leq 2^{-n+1}\right\} .
$$

It is obvious that $\left\{E_{n}\right\}_{0 \leq n<\infty}$ is a partition of $\Omega$ such that, for each $n, 0<$ $n<\infty$, there is $M>0$ with $1 / M \leq\|f(t)\| \leq M$, for all $t$ in $E_{n}$, and $f$ is zero on $E_{0}$. Let $\mu_{n}$ be the restriction of $\mu$ to $E_{n}$, and let $X_{n}=L^{p}\left(\mu_{n}, X\right)$. Then the partition $\left\{E_{n}\right\}$ induces an isometry $T$ from $L^{p}(\mu, X)$ onto $l^{p}\left(X_{n}\right)$, which is given by

$$
T(h)=\left\{\left.h\right|_{E_{n}}\right\}_{n \geq 1} \quad \text { for } h \text { in } L^{p}(\mu, X) .
$$

By Lemmas 4 and 5, $\left.f\right|_{E_{n}} /\left\|\left.f\right|_{E_{n}}\right\| \in \operatorname{str}-\exp B_{L^{p}\left(\mu_{n}, X\right)}$ whenever $\left.f\right|_{E_{n}} \neq 0$. By Lemma 6 we have $f \in \operatorname{str}-\exp B_{L^{p}(\mu, X)}$. Q.E.D.

\section{REFERENCES}

[DU] J. Diestel and J. J. Uhl, Jr., Vector measures, Math. Surveys, vol. 15, Amer. Math. Soc., Providence, RI, 1977.

[EV] G. Emmanuele and A. Villani, Lifting of rotundity properties from $E$ to $L^{p}(\mu, E)$, Rocky Mountain J. Math. 17 (1987), 617-627.

[G1] P. Greim, Strongly exposed points in Bochner $L^{p}$-spaces, Proc. Amer. Math. Soc. 88 (1983), 81-84.

[G2] _ A note on strong extreme and strongly exposed points in Bochner $L^{p}$-spaces, Proc. Amer. Math. Soc. 93 (1985), 65-66. 
[IT] A. Ionescu Tulcea and C. Ionescu Tulcea, Topics in the theory of lifting, Springer-Verlag, New York, 1969.

[J] J. A. Johnson, Strongly exposed points in $L^{p}(\mu, X)$, Rocky Mountain J. Math. 10 (1980), 517-519.

[S] M. Smith, Rotundity and extremity in $l^{p}\left(X_{i}\right)$ and $L^{p}(\mu, X)$, Contemp. Math., vol. 52, Amer. Math. Soc., Providence, RI, 1983, pp. 143-162.

Department of Mathematics and Statistics, Miami University, Oxford, OHio 45056

E-mail address: zhu@miavx1. acs .muohio.edu

Department of Mathematics, University of Iowa, Iowa City, Iowa 52242

E-mail address: bllin@math.uiowa.edu 